\title{
Asymmetric Interhemispheric Transfer in the Auditory Network: Evidence from TMS, Resting-State fMRI, and Diffusion Imaging
}

\author{
Jamila Andoh, ${ }^{1,2}$-Reiko Matsushita, ${ }^{2}$ and Robert J. Zatorre ${ }^{2}$ \\ ${ }^{1}$ Department of Cognitive and Clinical Neuroscience, Central Institute of Mental Health, Medical Faculty Mannheim/Heidelberg University, 68159 \\ Mannheim, Germany, and ${ }^{2}$ Montreal Neurological Institute, McGill University, Canada and International Laboratory for Brain, Music, and Sound Research, \\ Montreal, Quebec H3A 2B4, Canada
}

Hemispheric asymmetries in human auditory cortical function and structure are still highly debated. Brain stimulation approaches can complement correlational techniques by uncovering causal influences. Previous studies have shown asymmetrical effects of transcranial magnetic stimulation (TMS) on task performance, but it is unclear whether these effects are task-specific or reflect intrinsic network properties. To test how modulation of auditory cortex (AC) influences functional networks and whether this influence is asymmetrical, the present study measured resting-state fMRI connectivity networks in 17 healthy volunteers before and immediately after TMS (continuous theta burst stimulation) to the left or right AC, and the vertex as a control. We also examined the relationship between TMSinduced interhemispheric signal propagation and anatomical properties of callosal auditory fibers as measured with diffusion-weighted MRI. We found that TMS to the right AC, but not the left, resulted in widespread connectivity decreases in auditory-and motor-related networks in the resting state. Individual differences in the degree of change in functional connectivity between auditory cortices after TMS applied over the right AC were negatively related to the volume of callosal auditory fibers. The findings show that TMS-induced network modulation occurs, even in the absence of an explicit task, and that the magnitude of the effect differs across individuals as a function of callosal structure, supporting a role for the corpus callosum in mediating functional asymmetry. The findings support theoretical models emphasizing hemispheric differences in network organization and are of practical significance in showing that brain stimulation studies need to take network-level effects into account.

Key words: callosal auditory fibers; interleaved silent steady state; probabilistic tractography; resting-state fMRI; theta burst stimulation

\section{Significance Statement}

We investigated the lateralized functional networks involved in auditory processing and how modulation of the auditory cortex (AC) influences these networks. We demonstrated that transcranial magnetic stimulation (TMS) to the AC resulted in changes in connectivity in auditory and motor-related networks in the resting state. Such changes were strongly asymmetric, with stimulation of the right $\mathrm{AC}$ resulting in more widespread decreases in connectivity compared with stimulation of the left AC. Finally, we showed that individual differences in the degree of modulation of functional connectivity in AC were related to structural measures of the callosal auditory pathway. The findings support theoretical models emphasizing hemispheric differences in network organization; they also show that TMS studies need to consider network-level effects of stimulation.

\section{Introduction}

Auditory brain networks have repeatedly been shown to be functionally asymmetric in a wide variety of domains, notably in the

This work was supported by the Natural Sciences and Engineering Research Council to R.J.Z. and J.A., and an Erasmus Mundus ACN Exchange Network Grant to J.A. We thank M. Ferreira and I. Leppert for the development of the context of speech (Giraud et al., 2007) and tonal processing (Zatorre and Gandour, 2008), as also reflected in both local (Cha et al., 2014) and global (Morillon et al., 2010) functional interactions. However, the nature and significance of such asymmetries 
remain controversial (Poeppel, 2014). Noninvasive stimulation techniques can provide causal evidence about the involvement of targeted networks that can help to address these questions. Indeed, asymmetric patterns of behavioral outcome have been reported with transcranial magnetic stimulation (TMS) targeting auditory cortex (AC), with both language (Töpper et al., 1998; Andoh et al., 2006; Andoh and Paus, 2011) and tonal (Andoh and Zatorre, 2011) tasks.

Interpretation of TMS effects is complicated by the fact that they are not necessarily confined to the local area of stimulation because TMS-induced modulation influences interconnected brain regions via topographically organized pathways (Paus et al., 1997; Strafella et al., 2001). In particular, TMS applied over left temporal or frontal cortices during a language task increased task-related fMRI response in contralateral homologous areas (Thiel et al., 2006; Andoh and Paus, 2011). Similarly, TMS applied over right but not left AC increased neural activity in the contralateral AC during an auditory discrimination task (Andoh and Zatorre, 2013). Importantly, these interhemispheric propagation responses also depended on individual differences in interhemispheric interactions, such that higher functional connectivity (FC) between the two ACs was related to degree of TMS-induced contralateral recruitment (Andoh and Zatorre, 2013). Such interhemispheric interactions are likely mediated by transcallosal pathways (Johnston et al., 2008). Indeed, brain regions with greater structural interconnectivity tend to exhibit stronger interhemispheric FC (Greicius et al., 2009; Honey et al., 2009).

It is unclear whether stimulation-driven interhemispheric changes occur only in a task-specific context (perhaps as a compensation to preserve function) or whether they reflect intrinsic network properties. Resting-state fMRI is a useful tool to investigate neural organization in the absence of a cognitive task (for review, see Deco et al., 2011) and has recently been used to investigate network connectivity in response to TMS in the motor system (Cárdenas-Morales et al., 2014) and in cognitive control networks (Gratton et al., 2013) but has not been applied to auditory networks.

Here, we investigated the lateralized functional networks involved in auditory processing and how modulation of AC influences these networks. We used TMS to modulate activity and resting-state fMRI to identify the networks independently of task. We aimed to test the hypothesis that left and right ACs participate differently at the network level. Given their known asymmetries at local and global levels, we predicted that TMS targeting left versus right $\mathrm{AC}$ would result in different patterns of network activity. Additionally, we collected diffusion-weighted imaging data to test whether individual variation in interhemispheric anatomical connectivity might contribute to individual differences in the modulation of resting-state auditory networks. We reasoned that, if TMS-induced changes in FC are mediated by callosal pathways that interconnect the two ACs, then individual differences in anatomical connectivity in those pathways should be predictive of the magnitude of such effects.

\section{Materials and Methods}

Participants. Seventeen healthy volunteers ( 9 females) with normal hearing were recruited (mean \pm age, $23.1 \pm 4.9$ years). Participants were

Correspondence should be addressed to either Dr. Jamila Andoh or Dr. Robert J. Zatorre, Montreal Neurological Institute, 3801 University Street, Montreal, Quebec H3A 2B4, Canada, E-mail: jamila.andoh@zi.mannheim.de or robert.zatorre@mcgill.ca.

DOI:10.1523/JNEUROSCI.2333-15.2015

Copyright $\odot 2015$ the authors $\quad 0270-6474 / 15 / 3514603-10 \$ 15.00 / 0$ right-handed as assessed using the Crovitz and Zener handedness questionnaire (Crovitz and Zener, 1962). Participants spent on average 2.2 years in university ( $\mathrm{SD} \pm 2.8$ years) and had minimal formal musical training as measured by a self-reported history of musical experience, indicating $<1$ year of musical experience. Participants had no history of neurological disease or hearing impairment. All participants gave their informed consent, and the protocol was approved by the Montreal Neurological Institute Ethics Review Board. One participant (male) did not complete all sessions; these datasets were excluded from the fMRI and TMS analyses.

Experimental protocol. Volunteers were enrolled in three separate sessions on three different days. On each day, participants underwent resting-state fMRI scanning both before and after TMS. The target for continuous theta-burst stimulation (cTBS) stimulation on each day was either the right anterolateral area of Heschl's gyrus (HGal), the left HGal, or the vertex (as a control site); order of stimulation sites was counterbalanced across individuals.

Anatomical and diffusion MRI acquisition. MRI measurements were performed on a 3-T Siemens Trio scanner with a 32-channel head coil.

In each session on each day, high-resolution T1-weighted 3D images were acquired for each participant using a gradient echo sequence $(1 \mathrm{~mm}$ isotropic, TR/TE $=2300 / 2.98,192$ slices, matrix $=256 \times 256)$. To facilitate coregistration with the fMRI data, T1-weighted 3D images were acquired both before and after TMS

A separate diffusion-weighted image was also acquired in one of the three pre-TMS sessions. Diffusion encoding was achieved using a singleshot spin-echo echo-planar sequence with the following acquisition parameters: 64 slices, $\mathrm{b}=1000 \mathrm{~s} / \mathrm{m}^{2}, \mathrm{FOV}=256, \mathrm{TR} / \mathrm{TE}=8300 / 88$, GRAPPA parallel reconstruction, 99 directions, and a spatial resolution of $2 \times 2 \times 2 \mathrm{~mm}$.

fMRI acquisition. An interleaved silent steady state sequence (ISSS) was applied to acquire resting-state fMRI (Schwarzbauer et al., 2006). This acquisition sequence was used because it allowed resting state activity to be measured with reduced interference from the acoustic noise generated by gradient switching, a consideration of obvious importance when studying auditory networks. The scan consisted of a single run of 69 blocks. Each block was defined by 5 TR periods ( TR $=2.79 \mathrm{~s}$ ), with the first 3 TR periods consisting of silent slice-selective excitation pulses to maintain the steady state longitudinal magnetization (dummy scans), and the last 2 TR periods consisting of functional data acquisition (Fig. 1). The following imaging parameters were used: slice thickness, $3.5 \mathrm{~mm}$; number of slices, 36; field of view, $224 \mathrm{~mm}$; matrix size, $64 \times 64$; in plane spatial resolution $3.5 \times 3.5 \mathrm{~mm}$; acquisition bandwidth, $2442 \mathrm{~Hz}$; TR/ $\mathrm{TE}=2790 / 30 \mathrm{~ms}$. The duration of the run was $16.08 \mathrm{~min}$, resulting in 138 acquired volumes.

Field maps were also acquired for correction of distortions in the magnetic field, with a standard double-echo gradient echo field map sequence $(\mathrm{TE}=2.99$ and $5.45 \mathrm{~ms}, \mathrm{TR}=1000 \mathrm{~ms}$, matrix size $=64 \times 64)$ with 36 slices covering the whole head (voxel size $=3.5 \mathrm{~mm}$ isotropic). Participants were instructed to stay still with their eyes open while passively looking at a fixation cross. Visual monitoring equipment (SR Research EyeLink 1000) was used to ensure participants' awake state.

Localization of the stimulation sites. The auditory cortices were operationally defined as corresponding to the anterolateral Heschl's gyrus (respectively, R-HGal, L-HGal). Target locations were based on average coordinates (MNI 152 template) from our previous fMRI studies (Andoh and Zatorre, 2011, 2013) in which we used an auditory discrimination task. HGal was defined by spherical ROIs (radius $5 \mathrm{~mm}$ ) centered at $(54.6,-10.8,0.3)$ for R-HGal, and $(-51.4,-17.2,2.6)$ for the L-HGal. We used averaged coordinates to define $\mathrm{HGal}$ as our previous studies showed relatively small interindividual variability in location of functional responses. For each participant, the right and L-HGal target coordinates were transformed into the participant's native MRI space using the reversed MNI 152 template-to-native transformation matrix using FSL software. This registration procedure was performed after the first fMRI scan and before the start of TMS application. In addition, we also applied TMS over the vertex to control for nonspecific effects of TMS, such as acoustic and somatosensory artifacts. The vertex was defined 

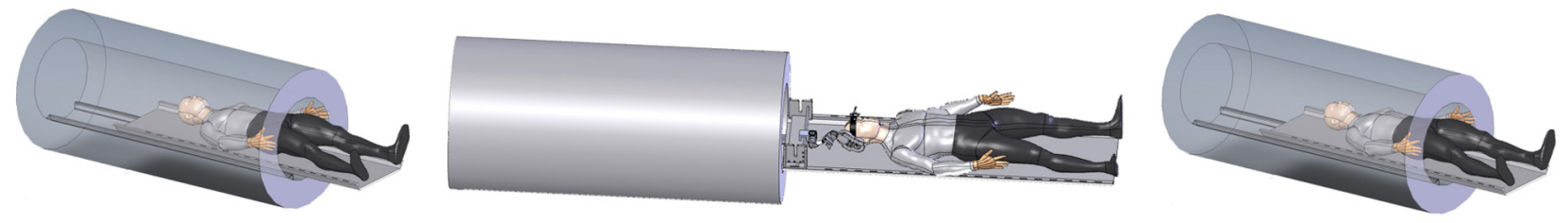

rS-ISSS
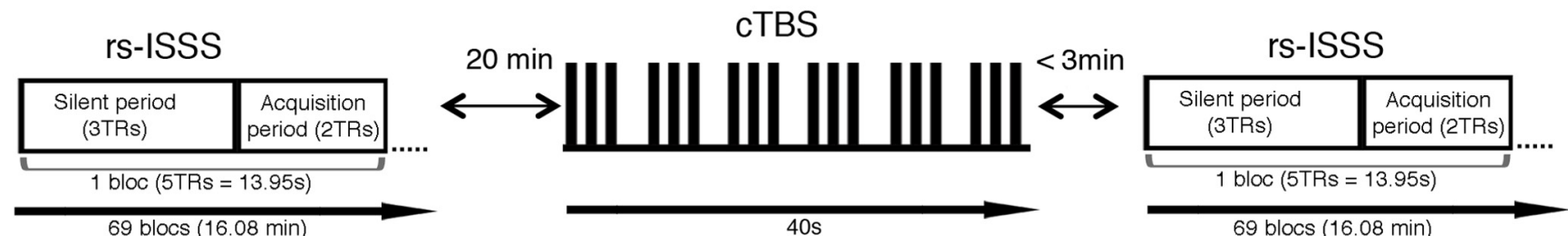

Figure 1. Experimental design. An ISSS was applied to resting-state fMRI (rs-ISSS) and consisted of successions of silent periods (3TRs) followed by an acquisition period (2TRs), with TR = 2.79s. Resting-state ISSS was performed before and after application of a continuous TBS protocol consisting of 3 pulses at $50 \mathrm{~Hz}(20 \mathrm{~ms}$ ) repeated every 200 ms (interstimulus interval) for a total duration of $40 \mathrm{~s}$ ( 600 pulses). The stimulated sites were the right and left anterolateral areas of Heschl's gyrus and the vertex performed on separate days and counterbalanced for order.

anatomically by the intersection between a sagittal line form the nasion to the inion and a coronal line from the tragus of both ears.

Combined fMRI and TMS protocol. A resting-state ISSS sequence was performed initially to map baseline (i.e., before any modulation induced by TMS) resting state connectivity. This was then followed by TMS application performed while the participants were lying down on the scanner bed, but outside of the bore of the magnet. TMS was delivered using an MR-compatible TMS coil (Magstim), and a Magstim Rapid2 stimulator, which was stationed in a room adjacent to the scanner. An infrared camera for online subject tracking and coil positioning (Polaris Spectra, NDI) was installed inside the scanner room, but at a safe distance from the MR magnet. A neuronavigation procedure was performed to guide the coil over the subject's scalp using Brainsight system (Rogue Research). A custom-designed MR-compatible jointed arm (Hybex Innovations) was used to mount the TMS coil in the scanner and to provide flexible positioning and rotation of the coil in multiple directions. TMS was applied with the coil held tangentially to the skull, with the handle pointing backward and parallel to the midline. cTBS was triggered using Presentation software (Neurobehavioral Systems) and consisted of three pulses at $50 \mathrm{~Hz}$ repeated at a frequency of $5 \mathrm{~Hz}$. Thus, a total of 600 pulses were delivered over a period of $40 \mathrm{~s}$ preceding the start of the post-cTBS fMRI acquisition. The stimulus intensities were set at $41 \%$ of the maximum stimulator output, which corresponds to $\sim 70 \%-80 \%$ of active motor threshold (Bestmann et al., 2003). TMS sessions were performed according to the published safety guidelines (Wassermann et al., 1996; Rossi et al., 2009).

The timing for starting the resting-state ISSS acquisition following cTBS was kept constant across participants and across days (Fig. 1) (for detailed methods and video procedure, see Andoh and Zatorre, 2012).

fMRI data analyses. Resting-state fMRI data were analyzed using Multivariate Exploratory Linear Optimized Decomposition into Independent Components (MELODIC) independent components analysis (ICA) from the FSL software package (Beckmann and Smith, 2004; Beckmann et al., 2005).

Preprocessing of fMRI data included motion correction, unwarping based on field map data within the MELODIC tool, high-pass temporal filtering (with a cutoff of $100 \mathrm{~s}$ ) and removal of nonbrain structures from the echo planar imaging volumes using Brain Extraction Tool (http://fsl. fmrib.ox.ac.uk/fsl/fslwiki/BET). The images were subsequently smoothed with a Gaussian kernel of FWHM of $5 \mathrm{~mm}$ and motion corrected. fMRI volumes were registered to the individual's structural scan using fMRIB's Linear Image Registration Tool (FLIRT) and to MNI-152 standard space images using fMRIB's Nonlinear Image Registration Tool (FNIRT) (http://fsl.fmrib.ox.ac.uk/fsl/fslwiki/FNIRT).

To avoid any biases, MELODIC ICA was applied using all fMRI scans together (i.e., fMRI pre-cTBS and post-cTBS over the right and L-HGal, and the vertex, corresponding to six fMRI scans per participant). Dual regression was then applied to each subject's preprocessed fMRI data to build subject-level versions of the group-ICA maps, and associated time- series (Beckmann et al., 2009). Specifically, for each subject (separately), the formerly obtained group-ICA spatial maps were used as spatial regressors against the subject's $4 \mathrm{D}$ fMRI data to estimate the resting state network time-series.

Differences in independent components maps pre-TMS versus post-TMS. To examine differences in independent components maps between fMRI scans pre-cTBS and post-cTBS, we proceeded in two steps: (1) for each of the three conditions (corresponding to the stimulated sites), withinsubject independent components maps differences were calculated between pre-cTBS and post-cTBS scans and merged across all participants; and (2) one-sample $t$ tests were performed separately for each merged within-subject independent components maps difference using 1000 permutations in randomize (fMRIB Software Library randomize version 2.9 ) with threshold-free cluster enhancement and family-wise error $(p<$ 0.05) (Nichols and Holmes, 2002). In addition, post hoc analyses were performed to examine the modulation of the different IC maps within the HG masks defined from standard maps provided by the HarvardOxford structural atlases (http://fsl.fmrib.ox.ac.uk/fsl/fslwiki/Atlases) using 1000 permutations in randomize (threshold-free cluster enhancement and family-wise error, $p<0.05$ ). This was performed to examine specific changes within the auditory network because that was our principal focus of interest.

Interhemispheric FC of auditory cortices. We extracted an index of FC between the right and the left HG as follows: HG was defined using an unbiased procedure from standard maps provided by the HarvardOxford structural atlases (http://fsl.fmrib.ox.ac.uk/fsl/fslwiki/Atlases) and transformed into each participant's native space using MNI-tonative transformation matrix using FSL software. For each participant and for each scan, we derived Pearson correlation coefficients calculated between the mean time courses of the BOLD signal from the right and the left HG using MATLAB (The MathWorks). These coefficients were then converted to $Z$ values through Fisher's $r$-to- $z$ transformation to obtain a normal distribution (Fisher, 1915). The result provides a specific quantitative index of the degree of interhemispheric functional interaction between left and right auditory cortices during the resting state scans.

Preprocessing of diffusion-weighted imaging data. Diffusion-weighted imaging data were preprocessed using FDT toolbox of FSL (fMRIB Software Library). Preprocessing included correction of artifacts induced by head motion and eddy currents, which was done by registering all the image volumes to the first b0 image via an affine transformation (Behrens et al., 2003). The preprocessed images were then fit to a diffusion tensor model at each voxel.

Probabilistic tractography. The BEDPOST tool (Behrens et al., 2003), which runs a Markov Chain Monte Carlo sampler, was used for building distributions of parameters describing the diffusion direction in each voxel. The outputs of the BEDPOSTX were used to compute the probabilistic diffusion tractography between the right and the left HG, using the corpus callosum as a waypoint mask by using Probtrackx in FSL (http://fsl.fmrib.ox.ac.uk/fsl/fslwiki/FDT). HG masks were defined using 

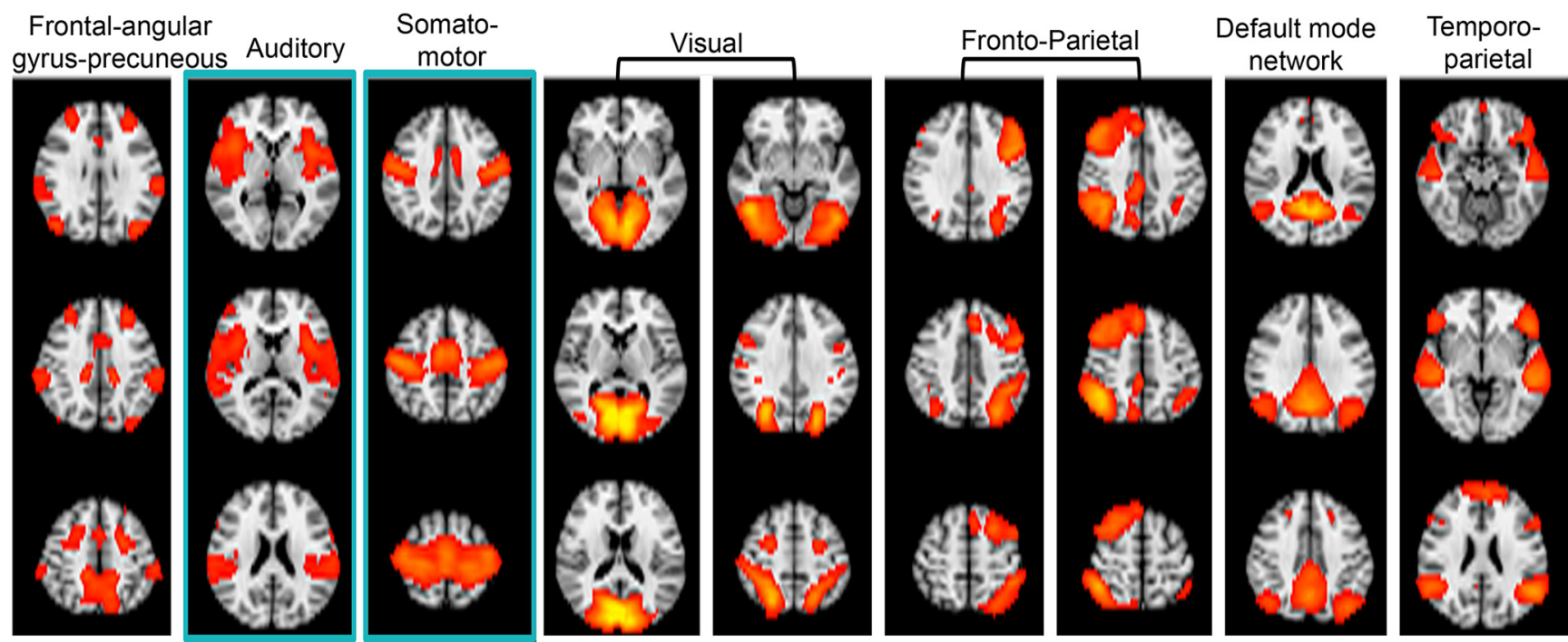

Figure 2. Identification of the main independent components from ICA. MELODICICA was applied using all resting-state ISSS fMRI scans (i.e., fMRI pre-cTBS and post-cTBS over the right and left $\mathrm{HGal}$, and the vertex). Columns represent independent components maps. The two networks that are modulated by TMS (auditory and somato-motor networks) are framed in blue.

standard maps available through the Harvard-Oxford atlas (see previous section), which are derived from structural data and segmentation. The corpus callosum was defined using the JHU white-matter tractography atlas (http://fsl.fmrib.ox.ac.uk/fsl/fslwiki/Atlases). The fiber tracking drew 5000 samples from each voxel in the seed mask. For each participant, we mapped the proportion of tracts starting from the right $\mathrm{HG}$ as a seed and crossing over to the contralateral hemisphere via the corpus callosum and entering the left HG target seed. We also mapped the symmetric tract (i.e., from the left to the right HG via the corpus callosum). Probabilities for connecting both seeds were normalized by thresholding each tract as a proportion of the number of streamlines (3\%) connecting both HGs. The threshold used was selected to avoid spurious results as the consequence of partial volume effect and image noise in the connectivity index. Next, we calculated the probability that either the path from the right to the left $\mathrm{HG}$ or from the left to the right HG passes through a certain voxel, which in terms of probability is given by the following:

$$
\begin{aligned}
p(r H G \rightarrow x \rightarrow l H G) \cup p(l H G \rightarrow x \rightarrow r H G) & \\
=p(r H G \rightarrow x \rightarrow l H G) & +p(l H G \rightarrow x \rightarrow r H G) \\
& \quad-p(r H G \rightarrow x \rightarrow l H G) \cap p(l H G \rightarrow x \rightarrow r H G) .
\end{aligned}
$$

This procedure provided a probability map between both gyri, which was used to extract FA measures and the number of streamlines. Each participant's FA image was transferred to the standard space using native-toMNI 152 template, and multiplied by the probability map obtained in the previous step. Then $f_{s} l$ stats (http://fsl.fmrib.ox.ac.uk/fsl/fslwiki/Fslutils) tool was used to extract the FA values and volume (i.e., the number of voxels $\times$ FA values) for non-zero voxels for each participant within the probability tract. The number of streamlines was calculated as follows: we multiplied the total number of voxels by the mean number of streamlines within each voxel within the probability tract between both gyri. FA volume and the number of streamlines provide complementary information of a fiber structure, with FA volume being an indicator of fiber density, and the number of streamlines reflecting the fiber volume.

To test whether the relation between TMS-induced changes in FC and interhemispheric structural connectivity was specific to Heschl's gyri, we performed tractography bilaterally in two other tracts: those interconnecting the planum temporale (PT), excluding tracts originating from $\mathrm{HG}$, and those interconnecting the primary visual cortices (V1), which was selected as a distant control region that we did not expect to have any relationship to FC in the auditory network. Tractography for bilateral PTs and V1s was performed using the same procedure as described above; PT tracts were defined using the Harvard-Oxford atlas, and V1 tracts were defined using the Jülich histological atlas (http://fsl.fmrib.ox. ac.uk/fsl/fslwiki/Atlases). We also calculated the number of streamlines and the FA values and volume within these tracts.

Regression analysis between measures of functional interhemispheric connectivity and structural measures of diffusivity. A regression analysis was performed to examine the relationship between the percent change in FC in the auditory network after TMS and three structural measures that we had hypothesized would be related (i.e., FA values), FA volume, and number of streamlines within the interhemispheric HG probability tract. For this analysis, we calculated \% FC change within the anatomically constrained HG because this structure was defined a priori based on anatomical criteria, and hence is free of any bias or circularity. The value corresponding to the percent change in FC [ $F C$ $(H G)$ post - FC (HG) pre)/FC (HG) pre], was then entered as a regressor with the FA values and FA volume within $\mathrm{HG}$, and with the number of streamlines for each participant, and for each condition (each of the three sites stimulated). To test for specificity of a relationship between \% FC change and structural measures related to HG interhemispheric connectivity, a similar analysis was performed between \% change in FC between bilateral HG after TMS and values and volume of FA and the number of streamlines within the probability tract of PT and V1.

\section{Results}

Timing between the end of the cTBS protocol and the subsequent resting-state ISSS acquisition was kept constant across participants and across days (resting-state ISSS scanning started at $2.8 \pm$ 0.5 min post-cTBS on day $1,2.5 \pm 0.3 \mathrm{~min}$ on day 2 , and $2.4 \pm 0.1$ min on day 3 ). The duration between the first and the second fMRI sessions was on average $5.8 \pm 4.7 \mathrm{~d}$, and the time between the second and the third fMRI session was on average $3.9 \pm 2.3 \mathrm{~d}$.

\section{Identification of resting-state brain networks}

MELODIC automatically estimated the number of independent components using a Laplacian approximation (Beckmann et al., 2009) (http://fsl.fmrib.ox.ac.uk/fsl). Thirteen independent component maps identified as artifacts were excluded, as voxels with high values within these ICs were mainly located in the CSF, WM, or large vessels, leaving a total of nine components (Fig. 2), which largely conform to expected networks identified in other studies (Beckmann et al., 2005; Damoiseaux et al., 2006; Smith et al., 2013). 
A rs-ISSS pre- minus post-cTBS aplied over the R-HGal
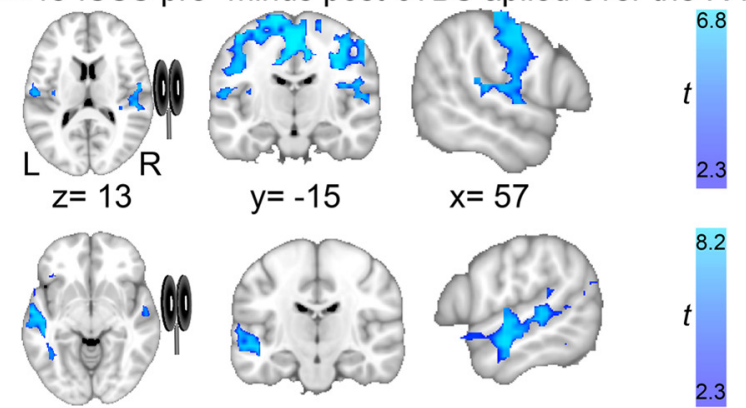

$z=-7$

$y=-18$

$x=-56$

B rs-ISSS pre- minus post-cTBS aplied over the L-HGal

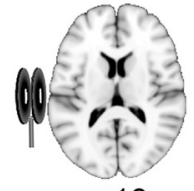

$z=13$

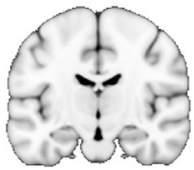

$y=-15$

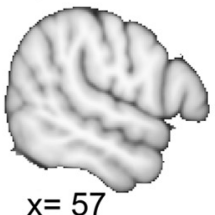

$x=57$
C rs-ISSS pre- minus post-cTBS aplied over the vertex

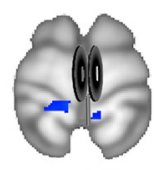

$z=73$

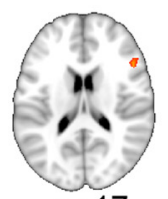

$z=17$

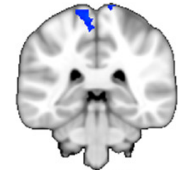

$y=-36$

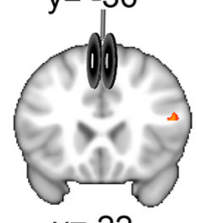

$y=22$

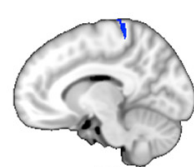

$x=-10$

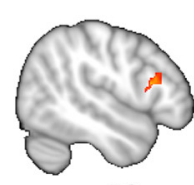

$x=50$
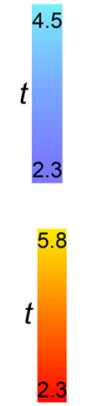

Figure 3. Differences between independent components maps pre-CTBS and post-cTBS applied over $(\boldsymbol{A})$ R-HGal showing the somato-motor network (top) and the auditory network (bottom), (B) the L-HGal, and $(\boldsymbol{C})$ the vertex for the somato-motor network (top) and the auditory network (bottom). Blue-light blue represents a decrease in FC after cTBS. Red-yellow represents an increase in FC after cTBS. IC maps differences were computed using 1000 permutations in randomize with threshold-free cluster enhancement and family-wise error $(p<$ 0.05 ) and represented with their respective $t$ values.

\section{Effects of cTBS on resting-state brain networks}

After cTBS applied over the R-HGal, we found significant decreases in FC specifically in those independent component maps related to auditory and motor networks (Fig. 3A; Table 1) and not in any of the others. These changes were observed both in ipsilateral and contralateral auditory regions, as well as in bilateral motor and premotor cortices, and in primary and secondary somatosensory cortices. We did not observe any increases in FC following stimulation. In contrast to the results after stimulation of the R-HGal, no significant change was found in FC in any of the independent component maps after cTBS applied over the L-HGal (Fig. 3B). To test whether there were any changes associated with L-HGal stimulation not picked up by the whole-brain analysis, we performed post hoc analyses using the anatomically defined HG ROIs as a mask. This analysis showed that cTBS applied over L-HGal did decrease FC, but only in the right (contralateral) HG (family-wise error-corrected, $p<0.05$ ).

When cTBS was applied over the vertex, we found a decrease in FC within the somato-motor network (bilaterally in the postcentral gyri), in the right superior parietal lobule, the left premotor cortex, and an increase in FC in the right inferior frontal gyrus
Table 1. Brain areas resulting from the comparison of independent components maps (somato-motor network and auditory network) pre-cTBS - post-cTBS applied over R-HGal ${ }^{a}$

\begin{tabular}{|c|c|c|c|c|c|}
\hline \multirow[b]{2}{*}{ Brain areas } & \multicolumn{3}{|c|}{ MNI coordinates (mm) } & \multirow{2}{*}{$\begin{array}{l}t \\
\text { values }\end{array}$} & \multirow{2}{*}{$\begin{array}{l}\text { Extent } \\
\text { (voxels) }\end{array}$} \\
\hline & $x$ & $y$ & $z$ & & \\
\hline \multicolumn{6}{|l|}{ IC map somato-motor network } \\
\hline \multicolumn{6}{|l|}{ Right hemisphere } \\
\hline Primary motor cortex & 1 & -25 & 53 & 6.8 & 81,311 \\
\hline Primary somatosensory cortex & 49 & -25 & 56 & 3.03 & \\
\hline Premotor cortex & 42 & -19 & 63 & 2.98 & \\
\hline Supramarginal gyrus & 49 & -33 & 41 & 3.73 & 15 \\
\hline Primary auditory cortex & 58 & -18 & 8 & 3.15 & 68 \\
\hline Secondary somatosensory cortex & 49 & -26 & 15 & 2.97 & \\
\hline Middle temporal gyrus & 71 & -24 & -2 & 2.76 & \\
\hline Heschl's gyrus & 50 & -16 & 9 & 2.70 & \\
\hline Superior temporal gyrus & 61 & -32 & 12 & 2.61 & \\
\hline Middle frontal gyrus & 49 & 18 & 40 & 2.67 & 54 \\
\hline \multicolumn{6}{|l|}{ Left hemisphere } \\
\hline Insular cortex & -35 & -13 & 9 & 4.28 & 202 \\
\hline Primary motor cortex & -43 & -16 & 42 & 5.54 & 27 \\
\hline Primary somatosensory cortex & -47 & -15 & 33 & 3.57 & \\
\hline Secondary somatosensory cortex & -55 & -15 & 12 & 3.41 & \\
\hline Premotor cortex & -39 & -18 & 63 & 2.57 & \\
\hline Heschl's gyrus & -55 & -17 & 9 & 2.59 & \\
\hline \multicolumn{6}{|l|}{ IC map auditory network } \\
\hline \multicolumn{6}{|l|}{ Right hemisphere } \\
\hline Middle temporal gyrus & 57 & -13 & -8 & 4.7 & 712 \\
\hline Superior temporal gyrus & 57 & -11 & -3 & 4.30 & \\
\hline Supramarginal gyrus & 49 & -34 & 9 & 4.28 & \\
\hline \multicolumn{6}{|l|}{ Left hemisphere } \\
\hline Superior temporal gyrus & -58 & -10 & -12 & 8.20 & 11,167 \\
\hline Inferior temporal gyrus & -42 & -54 & -4 & 3.64 & 1183 \\
\hline Frontal orbital cortex & -46 & 23 & -15 & 3.31 & 219 \\
\hline Inferior parietal lobule & -59 & -62 & 25 & 3.30 & 182 \\
\hline
\end{tabular}

Table 2. Brain areas resulting from the comparison of independent components maps for pre-cTBS - post-cTBS applied over the vertex in the somato-motor network and for post-cTBS - pre-cTBS applied over the vertex in the auditory network $^{a}$

\begin{tabular}{|c|c|c|c|c|c|}
\hline \multirow[b]{2}{*}{ Brain areas } & \multicolumn{3}{|c|}{$\begin{array}{l}\text { MNI coordinates } \\
(\mathrm{mm})\end{array}$} & \multirow{2}{*}{$\begin{array}{l}t \\
\text { values }\end{array}$} & \multirow{2}{*}{$\begin{array}{l}\text { Extent } \\
\text { (voxels }\end{array}$} \\
\hline & $x$ & $y$ & $z$ & & \\
\hline \multicolumn{6}{|l|}{ IC map somato-motor network } \\
\hline \multicolumn{6}{|l|}{ Right hemisphere } \\
\hline Primary somatosensory cortex & 6 & -42 & 76 & 4.22 & \multirow[t]{2}{*}{781} \\
\hline Superior parietal lobule & 5 & -44 & 78 & 4.33 & \\
\hline \multicolumn{6}{|l|}{ Left hemisphere } \\
\hline Primary somatosensory cortex & -17 & -37 & 74 & 3.90 & \multirow[t]{4}{*}{219} \\
\hline Primary motor cortex & -6 & -36 & 61 & 3.90 & \\
\hline Premotor cortex & -15 & -36 & 74 & 3.90 & \\
\hline Middle frontal gyrus & -38 & 18 & 32 & 5.33 & \\
\hline \multicolumn{6}{|l|}{ IC map auditory network } \\
\hline \multicolumn{6}{|l|}{ Right hemisphere } \\
\hline Inferior frontal gyrus (BA44) & 50 & 19 & 16 & 5.8 & 425 \\
\hline
\end{tabular}

${ }^{a}$ Coordinates are provided in MNI 152 space with their respective $t$ values.

within the auditory IC map network (Fig. 3C). No changes were observed within auditory cortices after stimulation of the vertex (Table 2).

Regression between interhemispheric FC and anatomical measures of diffusivity in transcallosal tracts

Figure $4 A$ shows the result of probabilistic tractography in bilateral HGs averaged across participants. Both HGs were interconnected through the posterior midbody of the corpus callosum, which is consistent with descriptions from other human neuro- 

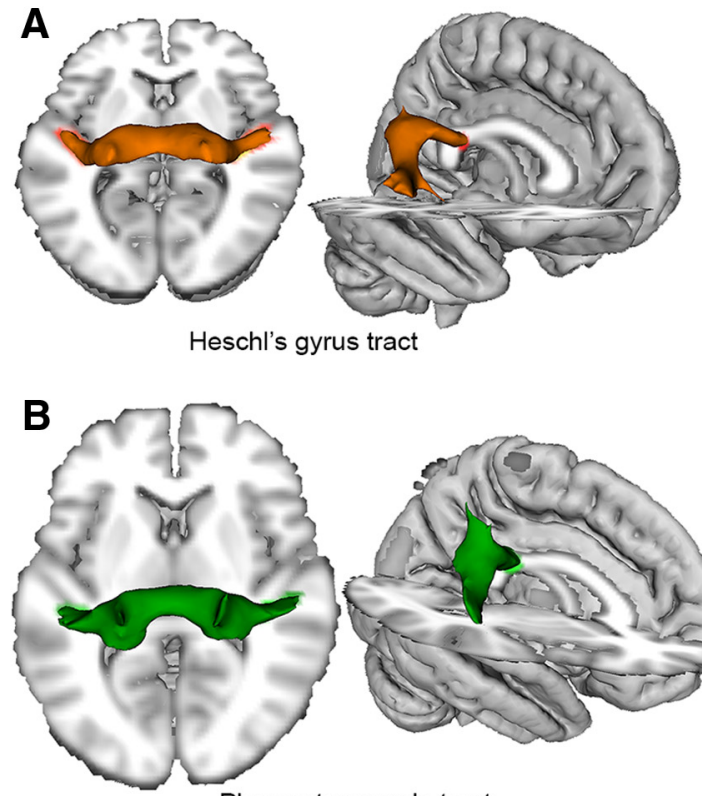

Planum temporale tract

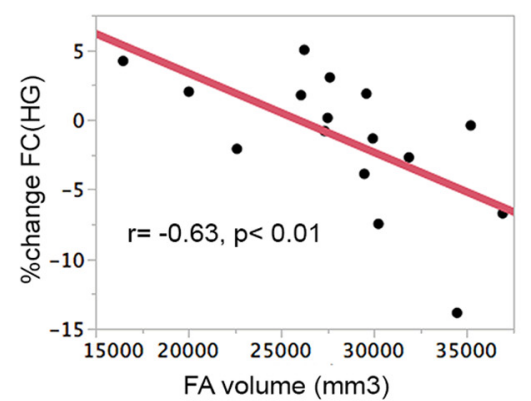

FA volume $(\mathrm{mm} 3)$
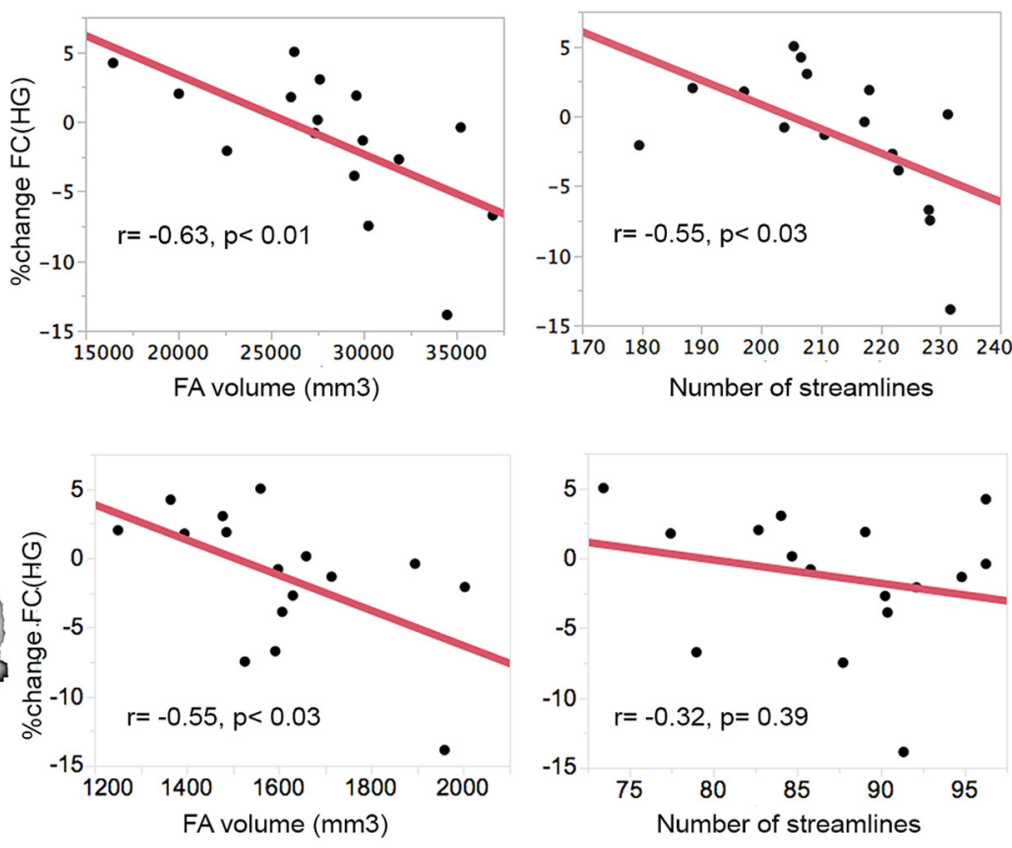

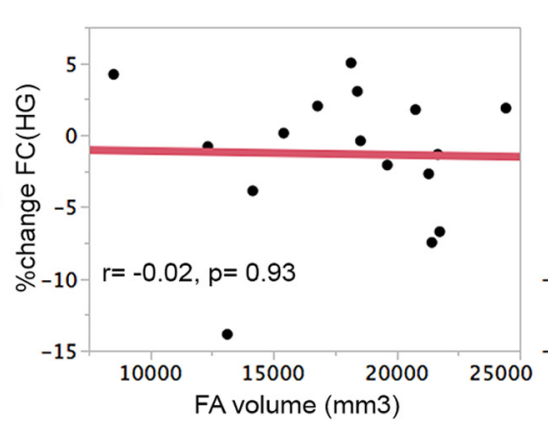

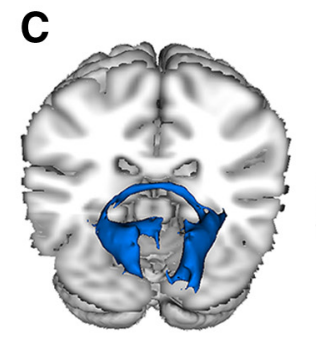

Visual cortex (V1) tract

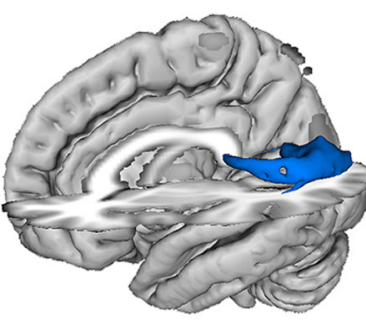

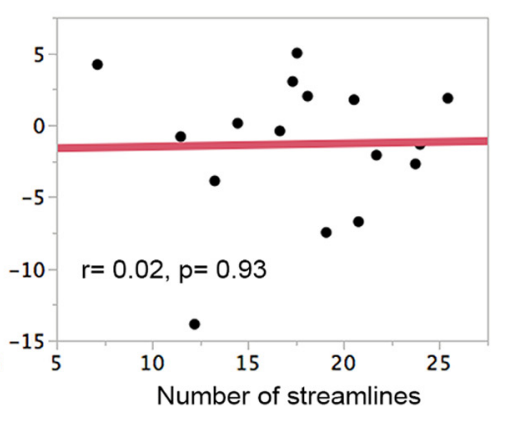

Figure 4. Regression analyses between the \% change in resting-state FC between left and right Heschl's gyri and FA volume and number of streamlines in the transcallosal Heschl's gyri tract ( $\boldsymbol{A}$ ), the transcallosal planum temporale tract $(\boldsymbol{B})$, and transcallosal primary visual cortices $(\boldsymbol{C})$.

imaging studies (Crippa et al., 2010; Javad et al., 2014) and with what might be expected based on anatomical tracer studies in other species (Demeter et al., 1990; Aboitiz et al., 1992). This lends validity to the tractography solution obtained.

The index of interhemispheric functional correlation for $\mathrm{HG}$ did not differ pre-cTBS for any of the 3 test days/conditions (one-way ANOVA, $\left.F_{(2,30)}<0.1, p=0.91\right)$. When we correlated the degree to which FC in each individual's HG changed following cTBS as a function of individual FA volume within the tract, we found a negative correlation following cTBS applied over the R-HGal $(r=-0.63, p<$ $0.01)$. The same relationship was seen as well with the number of streamlines $(r=-0.55, p<0.03)$, but not with FA values $(r=0.01$, $p=0.77$ ). Thus, those individuals with evidence of greater structural interhemispheric connectivity (greater FA volume or greater number of streamlines) were the ones who tended to demonstrate larger decreases in FC after stimulation (Fig. 4A). Similarly, we found a negative correlation between the percent change in FC in HG following cTBS applied over R-HGal and FA volume within the bilateral planum temporale tract $(r=-0.55, p<0.03)$ (Fig. $4 B)$, but not with the number of streamlines $(r=-0.32, p=0.39)$ or with FA values $(r<0.002, p=0.98)$.

Similar analyses using the bilateral V1 tract, used as a control, showed no correlation between the percent change in FC in HG after cTBS applied over R-HGal and FA volume within the bilateral V1 tract $(r=-0.02, p=0.93)$ or with the number of stream- lines $(r=0.02, p=0.93)$, or with FA values $(r=0.14, p=0.59)$ (Figure $4 C$ ). We also performed the same regression analyses by removing a potential "outlier" ( $\%$ FC change $=-13.87$; range, -13.87 to 5.05$)$; however, the results were still nonsignificant $(r<0.33, p=0.23)$.

Regarding cTBS applied over the L-HGal or the vertex, no correlations were found between the structural parameters (FA values and volume, number of streamlines) and the percent change in FC in $\mathrm{HG}(r<0.3, p>0.26)$.

\section{Discussion}

We demonstrated the following: (1) repetitive TMS to the AC results in changes to connectivity profiles in auditory and motorrelated networks in the resting state; (2) these changes are strongly asymmetric, such that stimulation of the right AC results in more widespread decreases in connectivity compared with stimulation of the left AC; and (3) individual differences in the degree of modulation of FC in AC are related to structural measures of the auditory transcallosal pathway, such that individuals with greater anatomical connectivity show greater TMS-induced changes in interhemispheric FC.

\section{Functional changes to resting-state networks}

We found that TMS modulated resting-state networks measured several minutes after the end of stimulation and that the changes 
consisted mostly of reductions in FC. These findings are coherent with prior observations that the inhibitory TMS protocol that we used (i.e., continuous theta burst stimulation) can induce longlasting inhibition of the underlying cortex (Huang et al., 2005; Nyffeler et al., 2006; Hubl et al., 2008). Our findings further show that network changes can be seen even in the absence of any overt task, which is consistent with previous studies showing changes in resting-state FC after TMS targeting primary somatosensory cortices or cerebellum (Halko et al., 2014; Valchev et al., 2015). The potential of TMS to modulate resting state networks is of interest because individual differences in resting connectivity can predict behavioral abilities (Baldassarre et al., 2012). In addition, TMS combined with resting-state fMRI may provide insight into the functional consequences of anatomical connections, in terms of inhibitory or excitatory interactions. For example, bilateral somatomotor cortices are positively correlated at rest (Fox et al., 2012), consistent with interhemispheric facilitation (Hanajima et al., 2001), whereas functional interactions between dorsolateral and ventromedial prefrontal cortex revealed by TMS suggest inhibitory mechanisms (Fox et al., 2005, 2012).

It is notable that stimulation of AC (on the right) resulted in changes to two specific resting-state networks: auditory and motor, both ipsilaterally and contralaterally. The modulation of auditory networks following AC stimulation is not unexpected, but modulation of motor networks is more surprising. This finding may reflect the important crosstalk between auditory and motor systems that has been noted in other contexts (Hickok et al., 2003, 2011; Zatorre et al., 2007). An important conclusion from these findings, and prior related ones (Strafella et al., 2001; Thiel et al., 2006) showing remote effects of TMS, is that interpretation of the behavioral effects of TMS should not be exclusively in terms of the site targeted, as is commonly suggested, but rather should take into account the distributed networks that may be affected by the stimulation.

Direct comparison of TMS effects in "active" (task-based) versus "resting" neural state have not been done, although there is evidence that baseline cortical excitability could influence postTMS excitability. For example, state-dependent effects of TMS during the resting state have previously been shown in the visual cortex, such that higher pre-TMS activity predicted greater postTMS activity (Pasley et al., 2009). In addition, motor or phosphene thresholds have been shown to be modulated by spatial attention (Bestmann et al., 2007) and motor training (Bütefisch et al., 2000).

Such findings could explain differences in TMS-induced effects reported between motor areas (usually stimulated at rest) and nonmotor brain areas, usually stimulated in relation with a cognitive task. State-dependent effects of TMS could also explain variability in TMS-induced effects within studies targeting similar areas because variability in task cognitive demands could result in different outcomes of TMS-induced effects (Bestmann et al., 2008; Ruff et al., 2008).

It is also well known that different TMS frequencies change the patterns of connectivity within a targeted network (Rounis et al., 2005; Eldaief et al., 2011). It seems highly likely that the topology of the network being targeted (e.g., balance of intrahemispheric and interhemispheric FC) would be a factor of variability for TMS parameters. It would be valuable to investigate whether networks with high interhemispheric connectivity (i.e., transcallosally mediated) are differentially modulated by different TMS frequencies.

\section{Asymmetry of TMS-induced FC changes in auditory network}

A salient aspect of these findings is the asymmetric response to stimulation of the auditory network, which seems independent of cognitive task because it appeared in resting-state networks. Specifically, FC within auditory and motor networks decreased significantly after TMS applied over the R-HGal, but only very little when TMS was applied over the L-HGal. Vertex stimulation also showed limited modulation of resting-state networks. The asymmetric response is related to our previous observation that FC between the two auditory cortices measured during an active melody discrimination task increased after TMS was applied over right but not L-HGal or vertex (Andoh and Zatorre, 2013). These findings are complementary because they show that TMS-induced effects in the auditory network are (1) neuroanatomically specific, (2) functionally asymmetric, and (3) statedependent because TMS-induced effects vary (increases or decreases in connectivity) depending on the neural state (i.e., involved during a cognitive task or not).

The hypothesis that hemispheric differences may exist in network properties has received support from various sources. Graph-theoretic metrics of anatomical connectivity show higher indices of interconnectivity as well as efficiency in the right hemisphere (Iturria-Medina et al., 2011). Similarly, measures of FC density show greater short- and long-range connectivity in the right than in the left superior temporal cortex (Tomasi and Volkow, 2012). Such asymmetric patterns suggest that the right hemisphere is better wired for the transfer of information across distant regions, as also suggested by functional asymmetries in resting-state networks. For example, Gotts et al., (2013) showed that left-hemisphere regions have greater preference for within-hemisphere interactions, whereas interactions in righthemisphere regions were more bilateral. Medvedev (2014) showed a stronger FC from the right to the left prefrontal cortex using near-infrared spectroscopy and Granger causality analysis, a measure providing directionality of connectivity. Right hemisphere asymmetry patterns have also been reported in the middle frontal and middle temporal gyri (Saenger et al., 2012), as well as in deeper structures, such as the anterior cingulate cortex (Yan et al., 2009). These observations are broadly consistent with our finding that stimulation of the right auditory cortex has much more widespread effects than similar stimulation on the left side.

It is possible that the asymmetric outcome observed could be related to different efficacy of TMS in the two hemispheres. A number of macroanatomical and microanatomical hemispheric differences in AC have been described (Penhune et al., 1996, Morosan et al., 2001, Hutsler and Galuske, 2003, Marie et al., 2015). How these asymmetric features interact with the consequences of TMS is unknown, but they may have contributed to our observations, as has been also suggested for asymmetries in MEG-evoked responses (Shaw et al., 2013). We therefore do not exclude the possibility that different coil orientations or stimulation parameters would lead to a greater influence of TMS over left AC than was evident here. However, given that intrinsic connectivity asymmetries are observed in the absence of stimulation, as described above, it seems unlikely that all of the TMS-induced asymmetry could be accounted for by these considerations.

The nature of information transfer from the right to the left hemisphere remains unclear but could be related to callosal structural properties as described below. Regardless of the underlying mechanism, the asymmetric outcome of TMS on restingstate activity suggests that previously reported asymmetric effects of TMS on behavior (Mottaghy et al., 1999; van Koningsbruggen et al., 2010; Andoh and Paus, 2011; Andoh and Zatorre, 2013) 
may need to be reinterpreted, taking into account the differential remote effects documented here when stimulation is applied over the left versus the right.

\section{Individual variability in interhemispheric structural and functional effects}

We found a significant relationship between the extent to which FC between auditory cortices changed after TMS-induced interference with the R-HGal, and tract volume of the structures connecting both auditory cortices. Specifically, participants showing decreased interhemispheric connectivity between auditory cortices following TMS showed greater volume in the tract interconnecting the auditory cortices. We also show specificity of the callosal auditory tract in mediating TMS-induced interhemispheric propagation, as no relationship was found for the control callosal visual pathways. We did observe a relationship between TMS-induced interhemispheric propagation and the tract volume of the planum temporale, but not with the number of streamlines, suggesting that the planum temporale shares some commonalities with callosal HG tracts but does not entirely overlap.

A similar relationship between interindividual differences in callosal anatomy and TMS-induced interhemispheric propagation of activity has previously been observed in the motor cortex using an interhemispheric inhibition paradigm (Wahl et al., 2007). However, in that case, differences in FA were found to predict the degree of interhemispheric interaction; whereas in the present study, FC in the auditory network was specifically related to the volume of the transcallosal auditory tract, and not to its microstructural integrity, as indexed by FA values. We used two complementary variables (i.e., number of streamlines and volume of the transcallosal auditory pathway), and both converged toward the same findings, such that the degree of FC correlated with both the volume and the number of streamlines within the transcallosal auditory tract. Such a relationship is not likely related to a higher white matter volume of the right HG because leftward asymmetries in the white matter volume of HG have previously been reported (Penhune et al., 1996; Morosan et al., 2001; Warrier et al., 2009). The somewhat different findings in motor and auditory systems may indicate intrinsic differences between modalities or may be related to the differential measures used (FC here, motor evoked potentials by Wahl et al., 2007), which may be sensitive to different aspects of callosal transmission.

Whether the corpus callosum plays an inhibitory or excitatory (facilitatory) role is still unclear (for review, see van der Knaap and van der Ham, 2011). Previous studies suggested that inhibitory and facilitatory transcallosal fibers may have different thresholds of activation (Ni et al., 2009; Voineskos et al., 2010). For example, the corpus callosum has been shown to have a facilitatory role at subthreshold TMS intensity and an inhibitory role at suprathreshold TMS intensity. Similarly, our previous and present work suggests that not only can the TMS intensity be a factor in variability of callosal modulation, but also anatomical features related to the organization of the callosal white matter fibers.

Our results underline the importance of individual variability in the connectivity between auditory cortices, measured both with functional and structural measures. Such findings are consistent with previous findings showing that interindividual variability in the interhemispheric auditory pathways was related to behavioral performance, such that stronger interhemispheric auditory pathways supported better information transfer (Wester- hausen et al., 2009). It is therefore important to take this into account for variability of TMS-induced effects.

In conclusion, the present findings show that TMS-induced interhemispheric signal propagation is transcallosally mediated, and neuroanatomically specific, supporting a role for callosal fibers in mediating functional asymmetry between homologous brain areas after external neural interference, such as TMS. Our findings show that auditory cortical functional networks are asymmetric, most likely reflecting differential patterns of interhemispheric and intrahemispheric connectivity. The findings support theoretical models emphasizing hemispheric differences in network organization; they are also of practical significance in showing that TMS studies need to consider network-level effects of stimulation.

\section{References}

Aboitiz F (1992) Brain connections: interhemispheric fiber systems and anatomical brain asymmetries in humans. Biol Res 25:51-61. Medline

Andoh J, Paus T (2011) Combining functional neuroimaging with off-line brain stimulation: modulation of task-related activity in language areas. J Cogn Neurosci 23:349-361. CrossRef Medline

Andoh J, Zatorre RJ (2011) Interhemispheric connectivity influences the degree of modulation of TMS-induced effects during auditory processing. Front Psychol 2:161. CrossRef Medline

Andoh J, Zatorre RJ (2012) Mapping the after-effects of theta burst stimulation on the human auditory cortex with functional imaging. J Vis Exp 67:e3985. CrossRef Medline

Andoh J, Zatorre RJ (2013) Mapping interhemispheric connectivity using functional MRI after transcranial magnetic stimulation on the human auditory cortex. Neuroimage 79:162-171. CrossRef Medline

Andoh J, Artiges E, Pallier C, Rivière D, Mangin JF, Cachia A, Plaze M, Paillère-Martinot ML, Martinot JL (2006) Modulation of language areas with functional MR image-guided magnetic stimulation. Neuroimage 29:619-627. CrossRef Medline

Baldassarre A, Lewis CM, Committeri G, Snyder AZ, Romani GL, Corbetta M (2012) Individual variability in functional connectivity predicts performance of a perceptual task. Proc Natl Acad Sci U S A 109:3516-3521. CrossRef Medline

Beckmann CF, Smith SM (2004) Probabilistic independent component analysis for functional magnetic resonance imaging. IEEE Trans Med Imaging 23:137-152. CrossRef Medline

Beckmann CF, DeLuca M, Devlin JT, Smith SM (2005) Investigations into resting-state connectivity using independent component analysis. Philos Trans R Soc Lond B Biol Sci 360:1001-1013. CrossRef Medline

Beckmann CF, Mackay CE, Filippini N, Smith SM (2009) Group comparison of resting-state fMRI data using multi-subject ICA and dual regression. 15th Annual Meeting of Organization for Human Brain Mapping, San Francisco, poster 441SU-AM.

Behrens TE, Woolrich MW, Jenkinson M, Johansen-Berg H, Nunes RG, Clare S, Matthews PM, Brady JM, Smith SM (2003) Characterization and propagation of uncertainty in diffusion-weighted MR imaging. Magn Reson Med 50:1077-1088. CrossRef Medline

Bestmann S, Baudewig J, Siebner HR, Rothwell JC, Frahm J (2003) Subthreshold high-frequency TMS of human primary motor cortex modulates interconnected frontal motor areas as detected by interleaved fMRI-TMS. Neuroimage 20:1685-1696. CrossRef Medline

Bestmann S, Ruff CC, Blakemore C, Driver J, Thilo KV (2007) Spatial attention changes excitability of human visual cortex to direct stimulation. Curr Biol 17:134-139. CrossRef Medline

Bestmann S, Swayne O, Blankenburg F, Ruff CC, Haggard P, Weiskopf N, Josephs O, Driver J, Rothwell JC, Ward NS (2008) Dorsal premotor cortex exerts state-dependent causal influences on activity in contralateral primary motor and dorsal premotor cortex. Cereb Cortex 18:1281-1291. CrossRef Medline

Bütefisch CM, Davis BC, Wise SP, Sawaki L, Kopylev L, Classen J, Cohen LG (2000) Mechanisms of use-dependent plasticity in the human motor cortex. Proc Natl Acad Sci U S A 97:3661-3665. CrossRef Medline

Cárdenas-Morales L, Volz LJ, Michely J, Rehme AK, Pool EM, Nettekoven C, Eickhoff SB, Fink GR, Grefkes C (2014) Network connectivity and indi- 
vidual responses to brain stimulation in the human motor system. Cereb Cortex 24:1697-1707. CrossRef Medline

Cha K, Zatorre RJ, Schönwiesner M (2014) Frequency selectivity of voxelby-voxel functional connectivity in human auditory cortex. Cereb Cortex. Advance online publication. Retrieved Sep 2, 2014. doi: 10.1093/ cercor/bhu193. CrossRef Medline

Crippa A, Lanting CP, van Dijk P, Roerdink JB (2010) A diffusion tensor imaging study on the auditory system and tinnitus. Open Neuroimag J 4:16-25. CrossRef Medline

Crovitz HF, Zener K (1962) A group-test for assessing hand- and eyedominance. Am J Psychol 75:271-276. CrossRef Medline

Damoiseaux JS, Rombouts SA, Barkhof F, Scheltens P, Stam CJ, Smith SM, Beckmann CF (2006) Consistent resting-state networks across healthy subjects. Proc Natl Acad Sci U S A 103:13848-13853. CrossRef Medline

Deco G, Jirsa VK, McIntosh AR (2011) Emerging concepts for the dynamical organization of resting-state activity in the brain. Nat Rev Neurosci 12:43-56. CrossRef Medline

Demeter S, Rosene DL, Van Hoesen GW (1990) Fields of origin and pathways of the interhemispheric commissures in the temporal lobe of macaques. J Comp Neurol 302:29-53. CrossRef Medline

Eldaief MC, Halko MA, Buckner RL, Pascual-Leone A (2011) Transcranial magnetic stimulation modulates the brain's intrinsic activity in a frequency-dependent manner. Proc Natl Acad Sci U S A 108:2122921234. CrossRef Medline

Fisher RA (1915) Frequency distribution of the values of the correlation coefficient in samples of an indefinitely large population. Biometrika 10: 507-521. CrossRef

Fox MD, Snyder AZ, Vincent JL, Corbetta M, Van Essen DC, Raichle ME (2005) The human brain is intrinsically organized into dynamic, anticorrelated functional networks. Proc Natl Acad Sci U S A 102:9673-9678. CrossRef Medline

Fox MD, Halko MA, Eldaief MC, Pascual-Leone A (2012) Measuring and manipulating brain connectivity with resting state functional connectivity magnetic resonance imaging (fcMRI) and transcranial magnetic stimulation (TMS). Neuroimage 62:2232-2243. CrossRef Medline

Giraud AL, Kleinschmidt A, Poeppel D, Lund TE, Frackowiak RS, Laufs H (2007) Endogenous cortical rhythms determine cerebral specialization for speech perception and production. Neuron 56:1127-1134. CrossRef Medline

Gotts SJ, Jo HJ, Wallace GL, Saad ZS, Cox RW, Martin A (2013) Two distinct forms of functional lateralization in the human brain. Proc Natl Acad Sci U S A 110:E3435-E3444. CrossRef Medline

Gratton C, Lee TG, Nomura EM, D'Esposito M (2013) The effect of thetaburst TMS on cognitive control networks measured with resting state fMRI. Front Syst Neurosci 7:124. CrossRef Medline

Greicius MD, Supekar K, Menon V, Dougherty RF (2009) Resting-state functional connectivity reflects structural connectivity in the default mode network. Cereb Cortex 19:72-78. CrossRef Medline

Halko MA, Farzan F, Eldaief MC, Schmahmann JD, Pascual-Leone A (2014) Intermittent theta-burst stimulation of the lateral cerebellum increases functional connectivity of the default network. J Neurosci 34:1204912056. CrossRef Medline

Hanajima R, Ugawa Y, Machii K, Mochizuki H, Terao Y, Enomoto H, Furubayashi T, Shiio Y, Uesugi H, Kanazawa I (2001) Interhemispheric facilitation of the hand motor area in humans. J Physiol 531:849-859. CrossRef Medline

Hickok G, Buchsbaum B, Humphries C, Muftuler T (2003) Auditorymotor interaction revealed by fMRI: speech, music, and working memory in area Spt. J Cogn Neurosci 15:673-682. CrossRef Medline

Hickok G, Houde J, Rong F (2011) Sensorimotor integration in speech processing: computational basis and neural organization. Neuron 69:407422. CrossRef Medline

Honey CJ, Sporns O, Cammoun L, Gigandet X, Thiran JP, Meuli R, Hagmann P (2009) Predicting human resting-state functional connectivity from structural connectivity. Proc Natl Acad Sci U S A 106:2035-2040. CrossRef Medline

Huang YZ, Edwards MJ, Rounis E, Bhatia KP, Rothwell JC (2005) Theta burst stimulation of the human motor cortex. Neuron 45:201-206. CrossRef Medline

Hubl D, Nyffeler T, Wurtz P, Chaves S, Pflugshaupt T, Lüthi M, von Wartburg R, Wiest R, Dierks T, Strik WK, Hess CW, Müri RM (2008) Time course of blood oxygenation level-dependent signal response after theta burst transcranial magnetic stimulation of the frontal eye field. Neuroscience 151:921-928. CrossRef Medline

Hutsler J, Galuske RA (2003) Hemispheric asymmetries in cerebral cortical networks. Trends Neurosci 26:429-435. CrossRef Medline

Iturria-Medina Y, Pérez Fernández A, Morris DM, Canales-Rodríguez EJ, Haroon HA, García Pentón L, Augath M, Galán García L, Logothetis N, Parker GJ, Melie-García L (2011) Brain hemispheric structural efficiency and interconnectivity rightward asymmetry in human and nonhuman primates. Cereb Cortex 21:56-67. CrossRef Medline

Javad F, Warren JD, Micallef C, Thornton JS, Golay X, Yousry T, Mancini L (2014) Auditory tracts identified with combined fMRI and diffusion tractography. Neuroimage 84:562-574. CrossRef Medline

Johnston JM, Vaishnavi SN, Smyth MD, Zhang D, He BJ, Zempel JM, Shimony JS, Snyder AZ, Raichle ME (2008) Loss of resting interhemispheric functional connectivity after complete section of the corpus callosum. J Neurosci 28:6453-6458. CrossRef Medline

Marie D, Jobard G, Crivello F, Perchey G, Petit L, Mellet E, Joliot M, Zago L, Mazoyer B, Tzourio-Mazoyer N (2015) Descriptive anatomy of Heschl's gyri in 430 healthy volunteers, including 198 left-handers. Brain Struct Funct 220:729-743. CrossRef Medline

Medvedev AV (2014) Does the resting state connectivity have hemispheric asymmetry? A near-infrared spectroscopy study. Neuroimage 85:400407. CrossRef Medline

Morillon B, Lehongre K, Frackowiak RS, Ducorps A, Kleinschmidt A, Poeppel D, Giraud AL (2010) Neurophysiological origin of human brain asymmetry for speech and language. Proc Natl Acad Sci U S A 107: 18688-18693. CrossRef Medline

Morosan P, Rademacher J, Schleicher A, Amunts K, Schormann T, Zilles K (2001) Human primary auditory cortex: cytoarchitectonic subdivisions and mapping into a spatial reference system. Neuroimage 13:684-701. CrossRef Medline

Mottaghy FM, Hungs M, Brügmann M, Sparing R, Boroojerdi B, Foltys $\mathrm{H}$, Huber W, Töpper R (1999) Facilitation of picture naming after repetitive transcranial magnetic stimulation. Neurology 53:1806-1812. CrossRef Medline

Ni Z, Gunraj C, Nelson AJ, Yeh IJ, Castillo G, Hoque T, Chen R (2009) Two phases of interhemispheric inhibition between motor related cortical areas and the primary motor cortex in human. Cereb Cortex 19:1654-1665. CrossRef Medline

Nichols TE, Holmes AP (2002) Nonparametric permutation tests for functional neuroimaging: a primer with examples. Hum Brain Mapp 15:1-25. CrossRef Medline

Nyffeler T, Wurtz P, Lüscher HR, Hess CW, Senn W, Pflugshaupt T, von Wartburg R, Lüthi M, Müri RM (2006) Repetitive TMS over the human oculomotor cortex: comparison of $1-\mathrm{Hz}$ and theta burst stimulation. Neurosci Lett 409:57-60. CrossRef Medline

Pasley BN, Allen EA, Freeman RD (2009) State-dependent variability of neuronal responses to transcranial magnetic stimulation of the visual cortex. Neuron 62:291-303. CrossRef Medline

Paus T, Jech R, Thompson CJ, Comeau R, Peters T, Evans AC (1997) Transcranial magnetic stimulation during positron emission tomography: a new method for studying connectivity of the human cerebral cortex. J Neurosci 17:3178-3184. Medline

Penhune VB, Zatorre RJ, MacDonald JD, Evans AC (1996) Interhemispheric anatomical differences in human primary auditory cortex: probabilistic mapping and volume measurement from magnetic resonance scans. Cereb Cortex 6:661-672. CrossRef Medline

Poeppel D (2014) The neuroanatomic and neurophysiological infrastructure for speech and language. Curr Opin Neurobiol 28:142-149. CrossRef Medline

Rossi S, Hallett M, Rossini PM, Pascual-Leone A (2009) Safety, ethical considerations, and application guidelines for the use of transcranial magnetic stimulation in clinical practice and research. Clin Neurophysiol 120:2008-2039. CrossRef Medline

Rounis E, Lee L, Siebner HR, Rowe JB, Friston KJ, Rothwell JC, Frackowiak RS (2005) Frequency specific changes in regional cerebral blood flow and motor system connectivity following rTMS to the primary motor cortex. Neuroimage 26:164-176. CrossRef Medline

Ruff CC, Bestmann S, Blankenburg F, Bjoertomt O, Josephs O, Weiskopf N, Deichmann R, Driver J (2008) Distinct causal influences of parietal versus frontal areas on human visual cortex: evidence from concurrent TMSfMRI. Cerebral cortex 18:817-827. CrossRef Medline 
Saenger VM, Barrios FA, Martínez-Gudiño ML, Alcauter S (2012) Hemispheric asymmetries of functional connectivity and grey matter volume in the default mode network. Neuropsychologia 50:1308-1315. CrossRef Medline

Schwarzbauer C, Davis MH, Rodd JM, Johnsrude I (2006) Interleaved silent steady state (ISSS) imaging: a new sparse imaging method applied to auditory fMRI. Neuroimage 29:774-782. CrossRef Medline

Shaw ME, Hämäläinen MS, Gutschalk A (2013) How anatomical asymmetry of human auditory cortex can lead to a rightward bias in auditory evoked fields. Neuroimage 74:22-29. CrossRef Medline

Smith SM, Beckmann CF, Andersson J, Auerbach EJ, Bijsterbosch J, Douaud G, Duff E, Feinberg DA, Griffanti L, Harms MP, Kelly M, Laumann T, Miller KL, Moeller S, Petersen S, Power J, Salimi-Khorshidi G, Snyder AZ, Vu AT, Woolrich MW, et al. (2013) WU-Minn HCP Consortium: resting-state $\mathrm{fMRI}$ in the Human Connectome Project. Neuroimage 80: 144-168. CrossRef Medline

Strafella AP, Paus T, Barrett J, Dagher A (2001) Repetitive transcranial magnetic stimulation of the human prefrontal cortex induces dopamine release in the caudate nucleus. J Neurosci 21:RC157. Medline

Thiel A, Habedank B, Herholz K, Kessler J, Winhuisen L, Haupt WF, Heiss WD (2006) From the left to the right: how the brain compensates progressive loss of language function. Brain Lang 98:57-65. CrossRef Medline

Tomasi D, Volkow ND (2012) Laterality patterns of brain functional connectivity: gender effects. Cereb Cortex 22:1455-1462. CrossRef Medline

Töpper R, Mottaghy FM, Brügmann M, Noth J, Huber W (1998) Facilitation of picture naming by focal transcranial magnetic stimulation of Wernicke's area. Exp Brain Res 121:371-378. CrossRef Medline

Valchev N, Ćurčić-Blake B, Renken RJ, Avenanti A, Keysers C, Gazzola V, Maurits NM (2015) cTBS delivered to the left somatosensory cortex changes its functional connectivity during rest. Neuroimage 1:386-397. CrossRef Medline

van der Knaap LJ, van der Ham IJ (2011) How does the corpus callosum mediate interhemispheric transfer? A review. Behav Brain Res 223:211221. CrossRef Medline

van Koningsbruggen MG, Gabay S, Sapir A, Henik A, Rafal RD (2010) Hemispheric asymmetry in the remapping and maintenance of visual saliency maps: a TMS study. J Cogn Neurosci 22:1730-1738. CrossRef Medline

Voineskos AN, Farzan F, Barr MS, Lobaugh NJ, Mulsant BH, Chen R, Fitzgerald PB, Daskalakis ZJ (2010) The role of the corpus callosum in transcranial magnetic stimulation induced interhemispheric signal propagation. Biol Psychiatry 68:825-831. CrossRef Medline

Wahl M, Lauterbach-Soon B, Hattingen E, Jung P, Singer O, Volz S, Klein JC, Steinmetz H, Ziemann U (2007) Human motor corpus callosum: topography, somatotopy, and link between microstructure and function. J Neurosci 27:12132-12138. CrossRef Medline

Warrier C, Wong P, Penhune V, Zatorre R, Parrish T, Abrams D, Kraus N (2009) Relating structure to function: Heschl's gyrus and acoustic processing. J Neurosci 29:61-69. CrossRef Medline

Wassermann EM, Grafman J, Berry C, Hollnagel C, Wild K, Clark K, Hallett M (1996) Use and safety of a new repetitive transcranial magnetic stimulator. Electroencephalogr Clin Neurophysiol 101:412-417. CrossRef Medline

Westerhausen R, Grüner R, Specht K, Hugdahl K (2009) Functional relevance of interindividual differences in temporal lobe callosal pathways: a DTI tractography study. Cereb Cortex 19:1322-1329. CrossRef Medline

Yan H, Zuo XN, Wang D, Wang J, Zhu C, Milham MP, Zhang D, Zang Y (2009) Hemispheric asymmetry in cognitive division of anterior cingulate cortex: a resting-state functional connectivity study. Neuroimage 47: 1579-1589. CrossRef Medline

Zatorre RJ, Gandour JT (2008) Neural specializations for speech and pitch: moving beyond the dichotomies. Philos Trans R Soc Lond B Biol Sci 363:1087-1104. CrossRef Medline

Zatorre RJ, Chen JL, Penhune VB (2007) When the brain plays music: auditory-motor interactions in music perception and production. Nat Rev Neurosci 8:547-558. CrossRef Medline 